\title{
Similarity consideration of structural bifurcation buckling
}

\author{
Y.-P. Zhao
}

\begin{abstract}
Many structural bifurcation buckling problems exhibit a scaling or power law property. Dimensional analysis is used to analyze the general scaling property. The concept of a new dimensionless number, the response number-Rn, suggested by the present author for the dynamic plastic response and failure of beams, plates and so on, subjected to large dynamic loading, is generalized in this paper to study the elastic, plastic, dynamic elastic as well as dynamic plastic buckling problems of columns, plates as well as shells. Structural bifurcation buckling can be considered when $\operatorname{Rn}(n)$ reaches a critical value.
\end{abstract}

\section{Ähnlichkeitsbetrachtung bei Knickproblemen mit strukturellen Verzweigungen}

Zusammenfassung Viele Knickprobleme mit strukturellen Verzweigungen weisen eine Skalierungseigenschaft oder Potenzgesetz-Abhängigkeit auf. Mittels einer Dimensionsanalyse wird die allgemeine Skalierungseigenschaft untersucht. Das Konzept einer neuen dimensionslosen Kennzahl, der Antwort-Zahl (response number), die der Autor für die dynamische Plastizierung und das Versagen von Balken,Platten, usw. unter großen dynamischen Lasten vorgeschlagen hat, wird in der vorliegenden Arbeit verallgemeinert und zur Untersuchung von statischen und dynamischen Problemen des elastischen und plastischen Krickens von Stäben, Platten und Schalen angewendet. Strukturelle Verzweigungen bei allgemeinen Knickproblemen treten auf, wenn die AntwortZahl einen kritischen Wert erreicht hat.

\section{List of symbols}

$B$ dimension in Fig. 2(a)

$c \quad \sqrt{E / \rho}$, elastic stress wave speed in a rod; $\sqrt{E /[\rho(1-v)]}$, elastic stress wave speed in spherical shell

$\mathrm{Ca}$ $\rho V_{0}^{2} / E$, Cauchy number

Received: 15. February 1999

\section{Y.-P. Zhao}

Laboratory for Nonlinear Mechanics of Continuous Media (LNM), Institute of Mechanics

Chinese Academy of Sciences

Beijing 100080, People's Republic of China

The author wishes to thank the financial support from National Natural Science Foundation of China and the foundation of the director of Institute of Mechanics, CAS.
De $\tau / T$, Deborah number

$\rho V_{0}^{2} / \sigma_{0}$ Johnson's damage number

$E \quad$ Young's elastic modulus

$G$ shear elastic modulus

$\bar{G} \quad$ reduced effective shear modulus

$H$ thickness

Ir $\quad \sigma_{0} \sqrt{l} / K_{\mathrm{IC}}$, Irwin number

$k$ radius of gyration

$K_{\text {IC }} \quad$ material fracture toughness

$l, L$ two characteristic dimensions of a structures

$m, M$ mass

$n$ positive real number

$R \quad$ radius

$\mathrm{Rn}$ response number for $n=2$

$\operatorname{Rn}(n)$ response number for a given $n$

$T$ period of harmonic external force

$V_{0} \quad$ initial impact velocity

$\alpha \quad$ semi-angle of circular arc

$\varepsilon \quad$ strain

$\lambda \quad E / E_{t}$

$v \quad$ Poisson's ratio

$\rho$ density

$\sigma_{0} \quad$ yield stress

$\tau \quad$ twisting stress, relaxation time

\section{Subscripts \\ $c, \mathrm{cr}$ critical \\ $e$ effective \\ $s$ secant \\ $t \quad$ tangent}

\section{1}

\section{Introduction}

It is clear that physical laws should not depend on the choice of units. They must therefore be expressed using relationships between quantities that do not depend on their arbitrary chance, i.e., dimensionless combinations of the variables. In general, for mechanical analysis of an engineering problem and particularly in setting up experiments, it is very important to select the dimensionless parameters correctly. Some dimensionless numbers often used in solid mechanics are summarized in the Appendix.

Dimensional analysis may be successfully applied in theoretical studies where a mathematical model of the problem is available, in the processing of experimental data, and also in the preliminary analysis of physical phenomena. What are some of the virtues of applications of similarity methods to problems in engineering 
mechanics? The following, in particular, have been found to be valuable in several important respects:

- to explore the fundamental behavior involved in a little-understood phenomenon;

- to obtain experimental data for quantitative evaluation of a particular theoretical analysis;

- to generate a functional relationship empirically to solve a general problem;

- to obtain quantitative data for use in prototype design problems, particularly when mathematical theory is overly complex or even nonexistent;

- to evaluate limitations for an expensive system already in existence.

By making dimensionless the governing equations, the present author has recently suggested a dimensionless number termed "response number-Rn" [1], it has been shown that this number is quite useful to study the dynamic plastic response and failure of structures such as beams, plates subjected to large dynamic loading. In the case of impulsive loading, the response number is expressed by

$\mathrm{Rn}=\frac{\rho V_{0}^{2}}{\sigma_{0}}\left(\frac{L}{l}\right)^{2}$,

where $\rho$ and $\sigma_{0}$ are, respectively, density and the uniaxial yield stress of the material subjected to impact velocity $V_{0}$, $L$ and $l$ are the two characteristic dimensions of the structure, respectively. For example, $L$ can be taken as half the span of a beam, radius of a circular plate or half width for a rectangular plate, and $l$ can be taken to be the thickness of the beam or plate, so $L$ is always much larger than $l$. From Eq. (1) we know that $\mathrm{Rn}$ is the product of the Johnson's damage number $\mathrm{Dn}=\rho V_{0}^{2} / \sigma_{0}$ and the square of half the slenderness ratio for a beam, the product of the damage number and the square of half the thinness ratio for a circular plate or membrane, a square plate as well as a rectangular plate loaded dynamically. The response number can also be considered the ratio of the inertia force of the impulsive loading to the plastic limit load of the structure. Three aspects has been reflected from this dimensionless number: the first one is the inertia of the applied dynamic loading, the second one is the resistance ability of the material to the deformation caused by the loading, and the last one is the geometrical influence of the structure on the dynamic response.

In the same manner, the response number for the elastic response of thin beams and plates loaded impulsively is, obviously, expressed as follows [1]

$\mathrm{Rn}=\frac{\rho V_{0}^{2}}{E}\left(\frac{L}{l}\right)^{2}$,

where $E$ is the Young's modulus of the material. At this case, the response number is the product of the Cauchy number $\mathrm{Ca}=\rho V_{0}^{2} / E$ and the square of half the slenderness ratio of the beam or half the thinness ratio of the plate.

Obviously, the above mentioned dimensionless number will have different forms under different loading conditions, or under different deformation conditions (such as elastic deformation and plastic one). Particularly, the power of the ratio $L / /$ may take different values for different kinds of structures, such as columns, plates, shells, etc. The main objective of the present paper is, by using dimensional analysis, to extend this response number $\mathrm{Rn}$ to the study of some elastic or plastic structural bifurcation buckling problems subjected to both static and dynamic loads, which all exhibit scaling. Here by scaling we mean that two measurable quantities depend upon each other in a power-law fashion.

\section{2}

\section{General property of structural bifurcation buckling}

Let us first consider the structural bifurcation buckling problem by dimensional analysis. For an homogeneous, isotropic, elastic material, the elastic properties can be defined by two constants, Young's modulus, $E$, and Poisson's ratio, $v$. Buckling, either elastic or plastic, is usually present in structural systems when one geometric dimension is of a smaller order than another dimension [2]; for example, slenderness ratios of columns and thinness ratios of plates. Suppose that two geometric parameters $L$ and $l$ are used to define the geometry of the structure, then the functional relationship of the elastic problem of structural bifurcation buckling is

$\sigma_{\mathrm{cr}}=F(E, v, L, l)$

By the Buckingham $\pi$-theorem, we have

$\frac{\sigma_{\mathrm{cr}}}{E}=f\left(v, \frac{l}{L}\right)$.

If, as is usually the case, the dependence on the Poisson's ratio is weak, then Eq. (4) can be expressed by

$\frac{\sigma_{\mathrm{cr}}}{E}=f^{\prime}\left(\frac{l}{L}\right) g(v)$.

Indeed, many structures fall into the class

$\frac{\sigma_{\mathrm{cr}}}{E} \propto\left(\frac{l}{L}\right)^{n}$

where $n$ is a positive real number. Equation (6) shows that many elastic bifurcation buckling problems exhibit scaling property, in other words, power law property.

In the same manner, the equivalent relationship for plastic buckling is

$\frac{\sigma_{\mathrm{cr}}}{\sigma_{0}} \propto\left(\frac{l}{L}\right)^{n}$

Equation (7) means that many plastic buckling problems, as elastic ones, exhibit scaling property.

For a rigid, perfectly dynamic plastic buckling problem of a structure subjected to uniformly distributed initial velocity $V_{0}$, the general function is

$V_{0}=F\left(\rho, \sigma_{0}, L, l\right)$.

Buckingham's $\pi$-theorem then gives

$\frac{\rho V_{0}^{2}}{\sigma_{0}}=f\left(\frac{l}{L}\right)$ 
Indeed, many structures exhibit the following scaling property

$\frac{\rho V_{0}^{2}}{\sigma_{0}} \propto\left(\frac{l}{L}\right)^{n}$.

For a dynamic elastic buckling problem of a structure under uniformly distributed initial velocity, the general functional relationship is

$V_{0}=F(\rho, E, v, L, l)$.

Buckingham's $\pi$-theorem gives the following form

$\frac{\rho V_{0}^{2}}{E}=f\left(v, \frac{l}{L}\right)$.

If the dependence of the problem on $v$ is weak, then we have

$\frac{\rho V_{0}^{2}}{E} \propto\left(\frac{l}{L}\right)^{n}$.

Equation (13) show that many dynamic elastic buckling problems, as dynamic plastic ones, also exhibit scaling property.

From Eqs. (6), (7), (10) and (13), it is very natural to define a dimensionless number similar to $\mathrm{Rn}$ in [1]. The generalized response number for dynamic plastic failure (response, tearing, buckling, etc.) of structures can be suggested as

$\operatorname{Rn}(n)=\frac{\rho V_{0}^{2}}{\sigma_{0}}\left(\frac{L}{l}\right)^{n}$.

In this case the response number is the product of the Johnson's damage number and the $n$-th power of half the slenderness or half the thinness ratio of the structure. For brevity, when $n=2$, we will simply use Rn instead of $\mathrm{Rn}(2)$. Generally speaking, the value $n$ is dependent upon the type of structures, that is to say, $n$ will take different value for columns, plates and shells. For elasto-dynamic behavior of structures, the response number equivalent to Eq. (2) is

$\operatorname{Rn}(n)=\frac{\rho V_{0}^{2}}{E}\left(\frac{L}{l}\right)^{n}$.

From Eq. (15) we know that the response number in this case is the product of the Cauchy number and the $n$-th power of half the slenderness or half the thinness of the structure.

For the plastic buckling problem of a structure under static load $\sigma_{\mathrm{cr}}$, then the response number equivalent to equation (14) is

$\mathrm{Rn}(n)=\frac{\sigma_{\mathrm{cr}}}{\sigma_{0}}\left(\frac{L}{l}\right)^{n}$.

Similarly, for the elastic buckling problem of a structure under static load, the response number is

$\mathrm{Rn}(n)=\frac{\sigma_{\mathrm{cr}}}{E}\left(\frac{L}{l}\right)^{n}$.

To sum up, the critical condition for structural bifurcation buckling problems can be simply expressed by

$\operatorname{Rn}(n)=$ const .
Some examples will be given blow to show the validity of the use of generalized response number $\operatorname{Rn}(n)$ to express the critical condition for structural bifurcation buckling problems.

\section{3}

\section{Some examples}

\section{1}

\section{Buckling under static load}

Euler (1744) first determined the critical axial unstable stress of a simply supported column, shown in Fig. 1, as

$\sigma_{e}=E\left(\frac{\pi k}{2 L}\right)^{2}$

where $k$ is the radius of gyration, and $2 L$ the length of the column. It is noted that equation (19) can be rearranged into

$\mathrm{Rn}=\frac{\sigma_{e}}{E}\left(\frac{L}{k}\right)^{2}=\frac{\pi^{2}}{4}$

Then the unstable condition af a simply supported column under compression is considered when $\mathrm{Rn}=2.47$.

The critical elastic stress for a spherical shell subjected to uniformly distributed exterior load $P_{e}$ is

$P_{e}=\frac{2 E}{\sqrt{3\left(1-v^{2}\right)}}\left(\frac{H}{R}\right)^{2}$,

where $H$ and $R$ are the thickness and the radius of the shell, respectively. Equation (21) can be rewritten into the form

$\mathrm{Rn}=\frac{p_{e}}{E}\left(\frac{R}{H}\right)^{2}=\frac{2}{\sqrt{3\left(1-v^{2}\right)}}$.

If $v=0.3$, then the critical response number is $\mathrm{Rn}=1.21$. The lowest stress for bifurcation of this spherical shell can be obtained by introducing effective Young's modulus $E_{e}$ and effective Poisson's ratio $v_{e}$, thus we have

$P_{e}=\frac{2 E_{e}}{\sqrt{3\left(1-v_{e}^{2}\right)}}\left(\frac{H}{R}\right)^{2}$,

or

$\operatorname{Rn}=\frac{P_{e}}{E_{e}}\left(\frac{R}{H}\right)^{2}=\frac{2}{\sqrt{3\left(1-v_{e}^{2}\right)}}$,

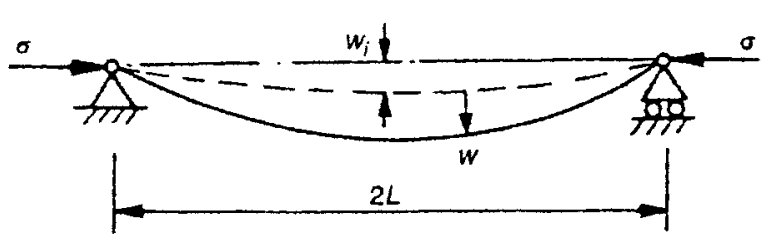

Fig. 1. Euler compressive bar 
where for Mises flow theory the effective Young's modulus and effective Poisson's ratio are determined by [3]

$\left\{\begin{array}{l}\frac{1}{E_{t}}=\frac{1}{E}\left[1+\frac{1}{4}\left(\frac{E}{E_{t}}-1\right)\right] \\ \frac{v_{e}}{E_{e}}=\frac{1}{E}\left[v-\frac{1}{4}\left(\frac{E}{E_{t}}-1\right)\right],\end{array}\right.$

where the tangent modulus $E_{t}$ is the function of $J_{2}$, then equation (22) is changed into

$\mathrm{Rn}=\frac{P_{e}}{E}\left(\frac{R}{H}\right)^{2}=\frac{4}{\sqrt{6(1+v)\left(1-2 v+\frac{E}{E_{i}}\right)}}$

The critical compressive stress for twisting bifurcation of a cruciform column as shown in Fig. 2(a) is [4]

$\sigma_{c}=G\left(\frac{H}{B}\right)^{2}$,

which can be written

$\mathrm{Rn}=\frac{\sigma_{c}}{G}\left(\frac{B}{H}\right)^{2}=1$,

where $G$ is the elastic shear modulus, and $H$ and $B$ are shown in Fig. 2(a). Equation (27) is obtained by using the flow theory. However, the bifurcation stress of the same structure by using the deformation theory is

$\bar{\sigma}_{c}=\bar{G}\left(\frac{H}{B}\right)^{2}$,

where $\bar{G}$ is a reduced effective shear modulus given by
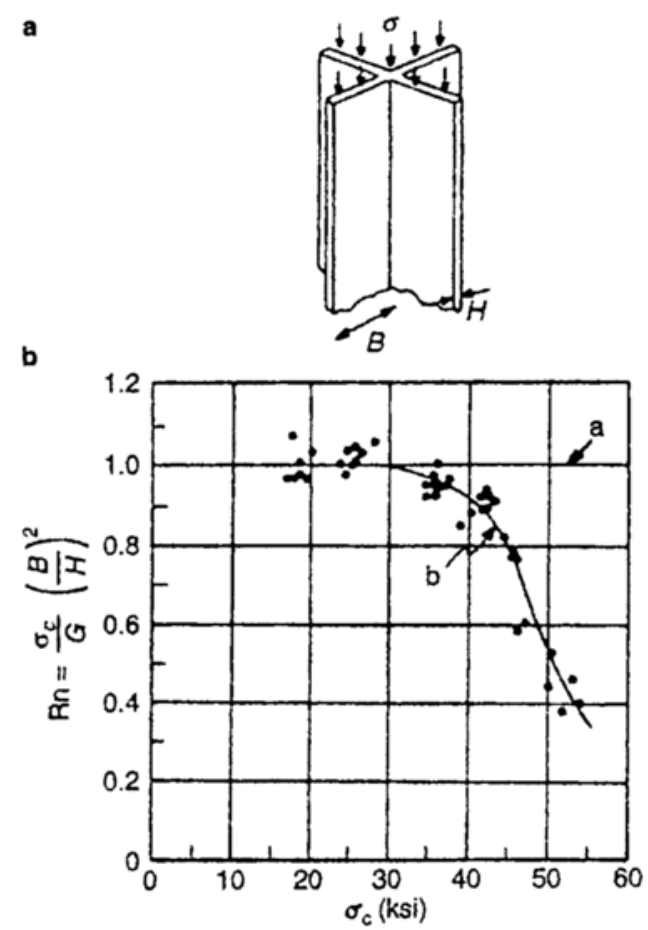

Fig. 2. a Cruciform column; $b$ Theoretical and experimental results for the plastic buckling of a cruciform column
$\frac{\bar{G}}{G}=\left[1+3 G\left(\frac{1}{E_{s}}-\frac{1}{E}\right)\right]^{-1}$

where $E_{s}=\sigma / \varepsilon$ is the secant modulus of the compressive stress-strain curve at $\vec{\sigma}_{c}$. Theoretical and experimental results for the plastic buckling of a cruciform column is shown in Fig. 2(b). These experiments were performed on specimens of 2024-T4 aluminum and the figure was taken from [5]. The discrepancy between the two theories for the cruciform column is more dramatic than occurs in most problems. Nevertheless, it is generally agreed that bifurcation-load predictions for plates based on $J_{2}$ deformation theory give reasonably good agreement with experimental buckling loads while predictions based on $J_{2}$ flow theory are consistently high [4].

The buckling stress of a simply supported circular arc with semi-angle $\alpha$ and radius $R$, as shown in Fig. 3, subjected to uniformly distributed radial pressure is

$\sigma_{\mathrm{cr}}=\frac{E H^{3}}{12\left(1-v^{2}\right) R^{3}}\left(\frac{\pi^{2}}{\alpha^{2}}-1\right)$,

where $H$ is the thickness of the arc, Eq. (31) can be rewritten into the form

$\operatorname{Rn}(3)=\frac{\sigma_{\mathrm{cr}}}{E}\left(\frac{R}{H}\right)^{3}=\frac{1}{12\left(1-v^{2}\right)}\left(\frac{\pi^{2}}{\alpha^{2}}-1\right)$

The buckling stress for a circular ring with radius $R$ and thickness $H$ subjected to uniformly distributed radial pressure is a special case of circular arc when $\alpha=\pi / 2$, i.e., we have

$\operatorname{Rn}(3)=\frac{1}{4\left(1-v^{2}\right)}$

for a complete circular ring under the condition of plane strain. The stress for plastic buckling for the same circular ring is

$\sigma_{p}=\frac{\pi}{4} \sigma_{0}\left(\frac{H}{R}\right)^{2}$.

It is obvious that equation (34) can be rearranged into

$\operatorname{Rn}=\frac{\sigma_{p}}{\sigma_{0}}\left(\frac{R}{H}\right)^{2}=\frac{\pi}{4}$.

The buckling stress of a axially compressed thin-walled cylindrical shell is

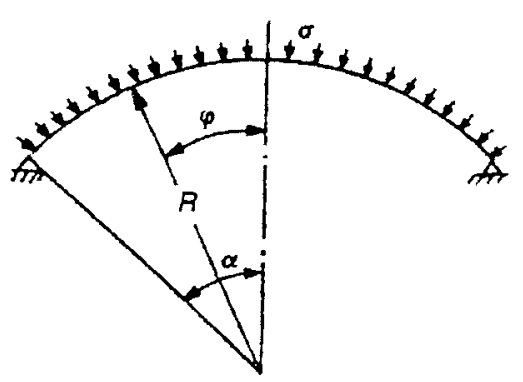

Fig. 3. Simply supported circular arc 
$\sigma_{\mathrm{cr}}=\frac{E H}{R \sqrt{3\left(1-v^{2}\right)}}$,

i.e.,

$\operatorname{Rn}(1)=\frac{\sigma_{\mathrm{cr}}}{E}\left(\frac{R}{H}\right)=\frac{1}{\sqrt{3\left(1-v^{2}\right)}}$.

The buckling twisting stress of this thin-walled cylindrical shell when $v=0.3$ is

$\tau_{\mathrm{cr}}=0.292 E\left(\frac{H}{R}\right)^{3 / 2}$,

i.e.,

$\operatorname{Rn}\left(\frac{3}{2}\right)=\frac{\tau_{\mathrm{cr}}}{E}\left(\frac{R}{H}\right)^{3 / 2}=0.292$.

Consider a simply supported circular plate subjected to a uniform radial stress $\sigma_{c}$, the elastic bifurcation stress for compressive loading obtained from the so called DonnellMushtari-Vlasov (DMV) criterion is

$\sigma_{c}=1.22 \frac{E}{1-v^{2}}\left(\frac{H}{R}\right)^{2}$,

or

$\mathrm{Rn}=\frac{\sigma_{c}}{E}\left(\frac{R}{H}\right)^{2}=\frac{1.22}{1-v^{2}}$

The dimensionless equation for axially compressed fully clamped circular plate is

$\mathrm{Rn}=\frac{\sigma_{c}}{E}\left(\frac{R}{H}\right)^{2}=\frac{0.35}{1-v^{2}}$

In the plastic range, the lowest bifurcation stress according to DMV theory can be obtain by using $E_{e}$ and $v_{e}$ instead of $E$ and $v$, for example, Eq. (42) is changed into

$\mathrm{Rn}=\frac{\sigma_{c}}{E_{e}}\left(\frac{R}{H}\right)^{2}=\frac{1.22}{1-v_{e}^{2}}$

where $E_{e}$ and $v_{e}$ are given by Eq. (25) for $J_{2}$ flow theory. For $J_{2}$ deformation theory,

$$
\left\{\begin{array}{l}
\frac{1}{E_{e}}=\frac{1}{4}\left(\frac{1}{E_{i}}+\frac{3}{E_{s}}\right) \\
\frac{v_{e}}{E_{e}}=-\frac{1}{4}\left[\frac{2\left(i-2 y^{\prime}\right)}{E}+\frac{1}{E_{\mathrm{t}}}-\frac{3}{E_{i}}\right] .
\end{array}\right.
$$

\section{2}

\section{Buckling under dynamic loading}

Slender copper rods dropped on to a rigid anvil buckle; the length of the waves created are the shorter the higher the speed of impact and the deformation is concentrated near to the impact end of the rod [6]. The stress to which such a rod is subjected at impact with speed $V_{0}$ is

$\sigma=\rho c V_{0}$,

where $c=\sqrt{E / \rho}$ is the elastic stress wave speed in the rod. Recalling the Euler buckling stress formula (19) for a simply supported strut of length $2 L$, then Eq. (19) is changed to the form as follows

$$
\left(\frac{2 L}{k}\right)^{4}=\pi^{4} \frac{E}{\rho V_{0}^{2}}
$$

which can be rewritten into the form of

$\operatorname{Rn}(4)=\frac{\rho V_{0}^{2}}{E}\left(\frac{L}{k}\right)^{4}=\frac{\pi^{4}}{16}$.

The dynamic elastic buckling condition of a complete spherical shell subjected to impulsive loading is presented by [7]

$\left(\frac{V_{0}}{c}\right)^{2}=\frac{2}{1+v}\left(\frac{H}{R}\right)^{2}$.

where $c^{2}=E /[\rho(1-v)], E q .(48)$ can be rearranged into

$\mathrm{Rn}=\frac{\rho V_{0}^{2}}{E}\left(\frac{R}{H}\right)^{2}=\frac{2}{(1+v)(1-v)}$.

The dynamic plastic buckling condition of a complete spherical shell to loaded impulsively is given by [7]

$\frac{\rho V_{0}^{2}}{\sigma_{0}}=\frac{2}{1-v}\left[\frac{\lambda}{5(1+v)}\right]^{1 / 2} \frac{H}{R}$,

where $\lambda=E / E_{t}$ is the ratio of the Young's and tangent moduli. Equation (50) can be rewritten into the form of

$\mathrm{Rn}(1)=\frac{\rho V_{0}^{2}}{\sigma_{0}}\left(\frac{R}{H}\right)=\frac{2}{1-v}\left[\frac{\lambda}{5(1+v)}\right]$.

The threshold initial impact velocity of a mass to cause the dynamic axial plastic buckling of a thin cylindrical shell, shown in Fig. 4, is given by [8]

$V_{0}^{2}=\frac{H \sigma_{0}}{\rho R\left(1+\frac{M}{m}\right)}$

i.e.,

$\operatorname{Rn}(1)=\frac{\rho V_{0}^{2}}{\sigma_{0}}\left(\frac{R}{H}\right)=\frac{1}{1+\frac{M}{m}}$,

where $m$ and $M$ are the mass of the shell and the flying block, respectively.
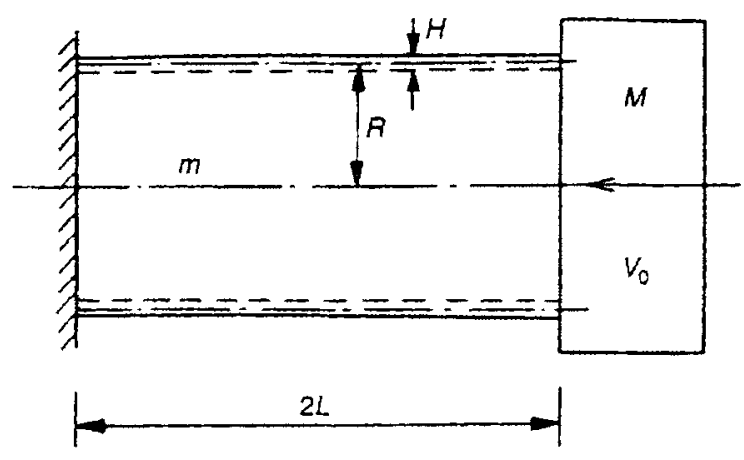

Fig. 4. Buckling of a cylindrical shell impacted axially by a mass 
Table 1. Dimensionless numbers in solid mechanics

\section{4}

\section{Discussion and concluding remarks}

Scaling (power-law) relationships have wide application in science and engineering, bifurcation buckling of structures is a good example. A very common view is that these scaling or power-law relations are nothing more than the simplest approximations to the available experimental data, having no special advantages over other approximations. It is not so. Scaling laws give evidence of a very deep property of the phenomena under consideration their self-similarity [9]. As a matter of fact, dimensional analysis has not be used previously to the analysis of bifurcation buckling problems.

This paper first analyzes, by using dimensional analysis, the general scaling property of structural bifurcation buckling problems, then this paper extends the application of response number Rn suggested in [1] to the analysis of structural bifurcation buckling problems. A generalized response number $R n(n)$ is suggested for elastic or plastic structural bifurcation buckling problems under static or dynamic loading. Some examples illustrate the validity of the use of this dimensionless number to represent the critical condition for structural bifurcation buckling.

\section{Appendix A}

\section{Some dimensionless numbers in solid mechanics}

Some dimensionless numbers in solid mechanics is given in Table 1. Similar to Irwin number in fracture mechanics, Zhao $[12,13]$ also suggested a dimensionless number $Z=E \sqrt{l} / K_{\mathrm{IC}}$, its application in fracture mechanics is discussed in detail in [12].

\section{References}

1. Zhao YP (1998) Suggestion of a new dimensionless number for dynamic plastic response of beams and plates. Arch. Appl. Mech., 68, 524-538

2. Chilver AH (1974) Design philosophy in structural stability. in Buckling of Structures (Ed. Budiansky, B.), Springer-Verlag, 331-345

3. Hutchinson JW (1974) Plastic buckling. in Advances in Applied Mechanics, 67-144

4. Hutchinson JW, Budiansky B (1976) Analytical and numerical study of the effects of initial imperfections on the inelastic buckling of a cruciform column. in Buckling of Structures (Ed. Budiansky, B.), Springer-Verlag, 98-105

5. Gerard G, Becker H (1957) Handbook of Structural Stability: Part I - Buckling of Flat Plates. Nat. Adv. Comm. Aeronaut. Tech. Note 3781

6. Johnson W (1972) Impact Strength of Materials. Edward Arnold

7. Jones N, Ahn CS (1974) Dynamic elastic and plastic buckling of complete spherical shells. Int. J. Solid Structures,10, 13571374

8. Jones N (1989) Structural Impact. Cambridge University Press

9. Barenblatt GI (1996) Scaling, self-similarity, and intermediate asymptotics. Cambridge University Press

10. Barenblatt GI (1993) Micromechanics of fracture. Theoretical and Applied Mechanics 1992, Bodner SR et al. (Eds.), Elsevier Science Publishers B. V

11. Zhao YP (1998) On the similarity methods in fracture mechanics. Forsch. Ingenieurwes., 64, 257-268

12. Zhao YP (1998) Prediction of structural dynamic plastic shear failure by Johnson's damage number. Forsch. Ingenieurwes., $63,349-352$

13. Zhao YP (1997) On some dimensionless numbers in fracture mechanics. Int. J. Fracture, 83, L7 $\sim$ L13 